\title{
Un écrit-témoin féminin: \\ 'Ma vie comme rivière' de Simonne Monet-Chartrand
}

\author{
Lise Ouellet \\ Life-Writing in the Feminine: \\ 'Ma vie comme rivière' \\ by Simonne Monet-Chartrand
}

Contemporary interest in the many forms of life writing constitutes a veritable 'marketplace in the experiential.' How are women positioned in this market? How do they negotiate this experience of the mirror and the consequent irruption of the public in the private? This study explores these questions with regards to the three volume autobiographical narrative of the left-wing, separatist, feminist activist, Simonne Monet-Chartrand. It focuses on the textual strategies adopted by the author, especially on the contract of narrator-narratee, on the configuration of the subject through the object of discourse, and the construction of a subject position as witness and model of social change for women. A woman writing a life: 'to be true to the very limit,' to write the unwritable intimate.

Ayant suscité depuis une vingtaine d'années l'adhésion du grand public en raison des témoignages touchants qu'ils révèlent, les écrits personnels ne cessent de se multiplier au point où la publication, la lecture et l'étude des journeaux intimes, des mémoires, des récits autobiographiques constituent actuellement un véritable 'marché du vécu'. ${ }^{1}$ Dans ce marché du vécu, quelle place les femmes occupentelles et de quelle façon vivent-elles l'expérience du miroir et celle de l'irruption du public dans le privé?

Comme d'autres femmes qui ont choisi de participer à l'ouverture de la sphère privée en communiquant leur histoire individuelle, Simonne Monet-Chartrand raconte dans les trois volumes de son récit autobiographique, Ma vie comme rivière (1919-1963) ${ }^{2}$, son enfance, son adolescence, son mariage, ainsi que son engagement politique (le Bloc populaire canadien, le Rassemblement pour l'indépendance 
nationale) et sa participation à plusieurs mouvements et associations (la Voix des Femmes du Canada, la Fédération des femmes). Le but de mon étude étant de dégager la spécificité de la prise de parole chez Simonne Monet-Chartrand, je discuterai d'abord du pacte narrateurnarrataire conclu par l'auteure, puis j'examinerai les traits saillants de l'écrit témoin et l'inscription du sujet dans son discours.

\section{Le pacte autobiographique: acte intime, acte social}

Longtemps condamnée à taire l'essentiel malgré son engagement social, Simonne Monet-Chartrand, à l'âge de soixante ans, abandonne en partie son 'travail bénévole d'écriture' (t. 1, p. 10) et tente d'avoir accès à son histoire, c' est-à-dire de 'découvrir les attitudes fondamentales' (t. 1, p. 3) de son passé. Cette quête d'identité s'accompagne d'une promesse de vérité et d'un désir de contestation inhérents à l'écriture autobiographique féminine: 'être vrai jusqu'au bout' (t. 1, p. 11) afin de dire ce qui ne se met pas sur la place publique, le vécu intime des femmes.

Si l'écriture de Ma vie comme rivière correspond à un profond besoin d'expression personnelle, elle $n^{\prime}$ en est pas moins liée au désir de l'Autre, comme le confirme la dédicace que l'on remarque dans le deuxième volet: 'A Michel, aux miens, au grand public. En toute amitié, $j$ 'offre (...) ces expériences que nous avons vécues fraternellement' (t. 2, p.3). Néanmoins, ce pacte atteste particulièrement un (legs à trois générations de femmes', ${ }^{3}$ puisque l'auteure compte par son témoignage faire revivre la condition fémininequébécoise des années trente jusqu'aux années soixante. D'ailleurs, la situation éditoriale ${ }^{4}$ de $M a$ vie comme rivière, $c^{\prime}$ est-à-dire le choix d'une maison d'édition féministe (les Éditions remue-ménage) et les propos de la couverture définissent une destinataire: 'Une lecture lucide et attentive des événements et des circonstances qui ont façonné notre destinée collective nous permettra d'impulser nos énergies comme nos imaginaires dans des formes plus précises et plus belles' (t. 1 et 2).

\section{La construction de l'écrit-témoin: de l'album de famille au collage socio-culturel}

Certes, le droit à raconter se justifie à plusieurs égards chez Simonne Monet-Chartrand puisqu' elle considère que son récit à des 'nuances près, [... lui] apparaît être le reflet de bien d'autres familles canadi- 
ennes-françaises de divers milieux, à diverses époques. ${ }^{5}$ L'auteure tient également à préciser qu'il ne s'agit pas d'une oeuvre littéraire, [mais ... $d^{\prime}$ ] une autobiographie basée sur les sentiments, [s]es sentiments devant une foule de choses ${ }^{\prime}{ }^{6}$. Aussi les rubriques du récit autobiographique classique ('je suis né, mon père et ma mère, la maison, le reste de la famille, le premier souvenir, le monde extérieur [etc.] $\left.]^{\prime}\right)^{7}$ se réaliseront-elles dans une forme spontanée et directe. C'est en ayant recours à des anecdotes, mais surtout à des textes authentiques, soit à caractère personnel tels que des extraits de son journal intime et de sa correspondance familiale, soit à caractère informatif comme ses conférences, ses scripts, ou bien encore à des photos la représentant elle-même, sa famille, des personnages connus, que Simonne MonetChartrand développe l'histoire de sa personnalité : le premier volet (1939-1942) ressemble à un album de famille et les deux autres (1939-1949, 1949-1963) à un collage socio-culturel construit selon les techniques du dossier de presse. La forme éclatée du récit autobiographique permet donc à la narratrice de trouver un équilibre entre la psycho-biographie 'l'individu se raconte à l'intérieur d'une trame événementielle' et l'ethnobiographie 'la personne est considérée comme le miroir de son temps, de son environnement. ${ }^{8}$

Se posant davantage comme témoin que comme écrivaine, Simonne Monet-Chartrand affirme avec raison 'qu'il est plus important et intéressant de lire des textes de fillette et d'adolescence ... pensés, ressentis au fur et à mesure, que d'avoir rédigé, à soixantedeux ans, une autobiographie subjective' ${ }^{9}$. De plus, selon la critique, Ma vie comme rivière constitue une 'copie non retouchée,' et la narratrice 'se garde bien d'interpréter (son passé) à la lumière de ce qu'elle est devenue. ${ }^{10}$ Néanmoins, dans la mesure où elle fait référence à vint-cinq années de son journal intime ou de sa correspondance et qu' elle communique une partie de ses souvenirs et de ses écrits informatifs, l'auteure a dû effectuer des remaniements, des coupures, des choix liés à sa situation actuelle, bref construire, infléchir le sens de son récit. Ainsi, la narration du premier volet de Ma vie comme rivière s'ouvre par une vaste prolepse qui précise les choix politiques de Simonne Monet-Chartrand et de son mari. En effet, c'est l'arrestation de Michel Chartrand pendant les événements d'octobre 1970 qui déclenche la quête d'identité propre au récit autobiographique: bouleversée par cet événement, la narratrice est retournée sur les lieux de son enfance à Beloeil où elle redécouvre la voix des siens et prend con- 
science d'être 'la vivante héritière d'une lignée d'hommes et de femmes de caractère, fiers et indépendants d'esprit : des dissidents politiques' (t. 1, p. 28) dont elle entend suivre les traces.

La suite du témoignage vise donc moins à donner une unité structurale, organique au passé de la militante qu'à dévoiler par des 'colliers' de souvenirs qui se juxtaposent 1 'émergence du moi social et les valeurs $\mathrm{du}$ ' 'je', à savoir celles de la transgression sociale, de la vérité, de la justice, du combat féministe et de l'amour éprouvé tant pour sa famille que pour certains groupes sociaux. Les anecdotes relatées couvrant l'enfance del'auteure présentent une fillette frêle et sensible mais qui s'échappe déjà à bicyclette pendant delongues heures ou qui se révolte entre autres contre des pratiques religieuses culpabilisantes:

Je n'aime pas les vicaires et les curés confesseurs, ni les confessionnaux. Là-dedans, il fait noir et ça ne sent pas bon. $Y$ aller toutes les semaines, $c^{\prime}$ est une corvée; ça me rend malade (...) Il faut toujours que je m'invente des péchés véniels (t. 1, p. 83).

De plus, si certaines parties du journal intime telles que 'Mon petit cahier journal' et 'L'âge ingrat' (1931-1933) fonctionnent comme une chronique évoquant les fêtes, les sorties, bref les événements de la vie quotidienne, ou peignent au contraire le 'je' de l'intérieur, c'est-à-dire les interrogations, les angoisses, la dépression liées à l'adolescence, $\mathrm{d}$ 'autres passages comme 'Chronique de couventine', ('Souvenirs de jeunesse étudiante' et 'Militantisme laïc') (1933-1940) décrivent notamment les injustices tolérées au couvent, la rencontre de Laure Gaudrault (institutrice engagée), les cours d'action sociale donnés par Soeur Marie Gérin-Lajoie et l'engagement de la jeune fille dans la JOC et la JEC. Par conséquent, l'intention militante qui traverse le récit $d^{\prime}$ enfance et d'adolescence annonce le contenu socio-politique des deuxième et troisième volets autobiographiques.

Quant au deuxième tome (1939-1942) construit par un 'jeu de stratification d'écrits de sources diverses [le journal de Simonne et celui de son père, la correspondance familiale, amicale et conjugale, les conférences et les mini-récits], d'événements ou de vécus analogues, ${ }^{\prime 1}$ il chevauche partiellement le premier volet, met l'accent sur le mariage et les grossesses de Simonne Monet-Chartrand et fait référence à des moments clés de la période : l'invasion de la Pologne, 
l'éclatement de la Deuxième Guerre mondiale (que l'auteure relate pendant son voyage à New York comme déléguée de la Fédération canadienne des étudiants catholiques), l'élection du governement libéral d'Adélard Godbout, la lutte contre la conscription, le droit de vote des femmes, la formation du Bloc populaire, la grève de l'amiante. Dans le troisième volet (1939-1963) se poursuit la 'petite histoire' socio-politique du Québec racontée par une mère de famille militante. Tout en élevant ses sept enfants, Simonne Monet-Chartrand participe à l'École des parents, écrit fréquemment des scripts pour Radio-Canada et fait partie de plusieurs associations et mouvements politiques qui reflètent son engagement et ses convictions : elle rencontre à plusieurs reprises les épouses des grévistes (d'Asbestos, de Thetford Mines, de Shawinigan, etc.) et est notamment membre de la Société Saint-Jean-Baptiste, des coopératives d'habitation et des caisses populaires, du Rassemblement pour l'indépendance nationale.

\section{Le temps de l'écrit-témoin: 'une inaltérable fidélité à soi-même'}

La critique a été sensible à Ma vie comme rivière, non pas parce que ce témoignage s'inscrit dans la Littérature, mais parce qu'il constitue un document autoréférentiel et socio-cultural fidèle aux valeurs de la narratrice. Selon Andrée Ferreti, 'il nous montre à l'oeuvre l'inaltérable fidélité à soi-même d'une femme dont le rythme intime ne change pas au cours des ans. ${ }^{12}$ Et Jean-Louis Major juge que ' $c$ ' est sans doute un indice de la vérité et de l'efficacité de ce récit autobiographique, ques'y maintiennent irréductiblement un engagement voué à la transformation sociale et une fidélité aux valeurs de continuité. ${ }^{13}$ Motivée par le désir de peindre la continuité de son vécu, Simonne Monet-Chartrand, structure $\mathrm{Ma}$ vie comme rivière par des 'rappels, de(s) retours, d(es) échos, de(s) répétitions, de(s) redondances, ${ }^{14}$ dont le flux s'apparente davantage au discours qu'au récit et dénote une perspective temporelle axée sur le présent et les temps du 'monde commenté'. En effet, bien que les formes verbales du 'monde raconté' (le passé simple, l'imparfait de l'indicatif et le conditionnel présent) existent dans $M a$ vie comme rivière, elles sont nettement subordonnées à celles du 'monde commenté' où prédominent le passé composé, le futur et le présent de l'indicatif. ${ }^{15}$

Ainsi le présent de la correspondance ou celui du journal intime permettent de livrer directement le vécu du personnage et de 
fusionner de façon subreptice le 'moi' d'autrefois et le 'je' du moment de l'écriture : 'Le 24 mai 1931.... On parle de mon âge ingrat. Je dois être faite comme ça. De toute façon, les grandes personnes ne sont jamais satisfaites de nous, les fillettes; $c^{\prime}$ est pourquoi j'essaie de faire à mon goût' (t. 1, p. 101). De plus, dans ce passage où la narratrice peint sa première communion, le présent de narration semble 'abol[ir] la distance entre le plan du récit et le plan du discours, et installer une instance de parole unique qui confond dans son ambivalence les divers ordres de la réalitét: ${ }^{16}$

Je dois gravir les deux marches d'accès à l'autel en baissant les yeux. Puis on me dit:

- Vos yeux doivent être fixés sur le Tabernacle. Le jour de la grande cérémonie, vous les fixerez sur l'Hostie. (...) Malgré toute mon application, la première hostie va se coller au fond de ma gorge. Je m'étouffe. La deuxième, je la transforme en boule. (t. 1 p. 78)

Enfin, par l'emploi généralisé des temps du discours, l'auteure semble créer un espace temporel, stable et rassurant, qui appartient à la fois au passé, à la situation actuelle et implicitement au futur:

J'ai vécu et je vis sous le signe de l'eau courante, mais à contrevent, à contre-courant. Ma Vie se situe et se déroule dans la ligne du risque. Essayer de bâtir une société socialiste, $c^{\prime}$ était et c'est encore aller au devant des difficultés (t. 1, p. 41). ${ }^{17}$

Les notions de passé / présent / futur s'interpénétrant dans Ma vie comme rivière provoquent donc un effet de vécu et d'instantanéité, puisque 'le lecteur est comme devant un reportage à la télévision. Tout ce dont on lui parle appartient à un monde où il vit lui-même, et non au monde du papier et de l' écriture' ${ }^{\prime 8} \mathrm{Il}$ me paraît peu important de trancher si cette perspective temporelle propreau document vécu et au témoignage est le fruit de certains remaniements ou, au contraire, si elle correspond à une 'copie conforme'. Grâce à l'omniprésence du présent et des temps du discours, la narratrice peint l'aspect inaltérable de son moi plutôt que ses déchirures ou ses transformations et produit par le fait même un texte qui adhère à son projet d'écriture : un récit encourageant dont 'les pensées, expéri- 
ences, action et souvenirs (...) évoqués (visent) à faciliter chez les lectrices et les lecteurs, des relations interpersonelles chaleureuses' (t. 3, p.7).

\section{La voix $\mathrm{du}$ ' je' témoin existe-t-elle?}

Désirant avant tout transmettre le 'témoignage d'une merveilleuse aventure de re-communication avec des personnes connues et aimées durant (sa) Vie' (t. 1, p.5), Simonne Monet-Chartrand livre également un récit autobiographique 'centré non sur l'émetteur mais sur le récepteur'. ${ }^{19}$ Ma vie comme rivière semble en effet traversé par les voix des modèles identificatoires (les parents, le mari, les amis de l'auteure, certaines personnalités, entre autres Thérèse Casgrain, Lionel Groulx, André Laurendeau, Louise Simard, Gabrielle Roy) qui définissent $l$ 'identité intime, sociale et idéologique du ' je'. Dans la mesure où la narratrice cède fréquemment la parole à l'Autre en relatant des anecdotes à caractère politique et en reproduisant notamment des extraits du Daily Journal de son père, de la correspondance amoureuse de ses parents, des lettres adressées par le public, des discours syndicaux de Michel Chartrand, ne pourrait-on pas affirmer que la propre voix du 'je' risque d'être étoufée par la création d'un système de voix qui se juxtaposent, se fragmentent et se répondent?

Pourtant, bien que diffuse, la voix du 'je' me paraît en fait omniprésente dans Ma vie comme rivière, car les 'effets de citation' ou de 'vécu' que l'on remarque sont orchestrés par la narratice. Ainsi dans le récit sur la 'campagne des conscrits', Simonne Monet-Chartrand semble de prime abord extérieure à son discours. En raison des informations 'objectives' qu'elle livre (dates, lieux, noms des candidats) et des techniques qu' elle emploie (le discours direct, le retour de la troisième personne, les tournures impersonnelles, la voix passive), les événements semblent se raconter d'eux-mêmes. Cependant grâce à l'opposition des propos du général Laflèche et de Michel Chartrand, la narratrice exprime ses idées 'sans les avoir dites, ${ }^{\prime 20} c^{\prime}$ 'est-à-dire qu'elle dévoile implicitement le clivage social qui existe entre 'eux', les fédéralistes, et 'nous', les Québécois nationalistes:

Mes amis, je suis un vieux soldat qui a fait deuxgrandes guerres. Notre premier devoir est d'assurer la défense du Canada (...) Le Canada est le grenier et l'arsenal des démocraties attaquées par 
l'ennemi allemand. (...) J'ai marché et je continuerai de marcher par la croix et par l'épée.

Votons bravement pour Drapeau en nous souvenant du sort que la GRC vient de faire à Marc Carrière. Souvenez-vous qu'Honoré Mercier, chef provincial nationaliste, a été élu parce que le peuple se souvenait de l'exécution de Louis Riel.

De plus, profondément antimilitariste, Simonne Monet-Chartrand intercale dans son journal intime écrit en 1939 des propos de son père qui sont étroitement liés à ses convictions:

14 octobre 1914 (...) Je ne sais si leurs chiffres sont exacts mais le gouvernement conservateur canadien désire recruter ici trentedeux-mille hommes, dans un jeune pays de huit millions d' habitants. C'est énorme. Combien y aura-t-il de morts? (t. 2, p. 45)

Le vécu intime du ' $\mathrm{je}$ ', en d'autres termes ses sentiments, ses espérances et sa vision du couple sont également en partie traduits par la voix de l'Autre. En effet, le 30 juillet 1917, Berthe Monet écrit à son mari que 'dans un frisson, étroitement enlacés, [ils] sentiron[t] couler l'oubli d'hier en même temps que l'espérance en un demain toujours radieux' (t. 2, p. 33). Et le 17 février 1952, Simonne MonetChartrand confie à Michel Chartrand qu'en rêve et en fait, (elle a) revu le merveilleux film de [leurs] années de vie commune : [leurs] envolées dans le temps et l'espace' (t. 3, p. 100).

Partageant les mêmes idéaux que son mari, la jeune femme n'en juge pas moins sa vie difficile, voire pénible à bien des égards, puisqu'en raison de l'engagement syndical de Michel Chartrand, elle élève la plupart du temps seule leurs enfants dans des conditions matérielles parfois fort précaires. Dans la correspondance conjugale, Simonne Monet-Chartrand exprime avec beaucoup de tact mais de façon très efficace son désir d'équilibrer la relation du couple et $\mathrm{d}^{\prime}$ affirmer davantage son 'moi'. Par des phrases que l'on pourrait qualifier 'd'expositives-verdictives', ${ }^{21}$ le 'je' rappelle notamment à son mari l'importance de certaines responsabilités familiales ou d'activités qu'elle juge essentielles:

Nous avons causé l'autre nuit ensemble, mais je tiens à y revenir. 
Mais pourquoi serais-je la seule gardienne de ce bien précieux? (leur bonheur) (t. 2,p. 271)

$\mathrm{J}$ 'ai comme un problème de conscience à refuser de participer aux cours du Service de préparation au mariage, aux réunions des équipes de foyers ou aux conférences de l'École des parents (t. 2, p. 211-212).

Désirant que Michel Chartrand soit davantage présent, l'épistolière ne demande pas directement á son mari de modifier sa conduite. Néamoins, elle affirme discrètement son autorité en portant un jugement sur des attitudes générales ou en s'incluant dans un 'nous' désireux de modifier 'à deux' une situation difficile et inquiétante surtout pour elle.

Cette manière masculine de penser est erronée et mène à des irresponsabilités de la part des maris et des pères. Notre avenir est fragile. Il faut l'analyser, l'étudier et le protéger. [...] Il nous faut plus de discipline, d'équilibre à l'intérieur de toutes nos tâches pour un meilleur épanouissement de notre jeunesse ardente et amoureuse (t. 2, p. 271).

Révélant ses souffrances, ses expériences et son désir de vivre, la voix du témoin vise à faire entendre celle de la 'majorité oubliée', $c^{\prime}$ est-à-dire celle des femmes de 'tous milieux' et de 'tous âges' (t.3, p. 14): la voix du 'je' et celle des autres femmes semblent donc se répondre, se rejoindre, se fondre dans Ma vie comme rivière. Si la narratrice, par la correspondance et les anecdotes qu'elle présente, reconnaît de grandes écrivaines telles que Gabrielle Roy, ou des femmes dévouées comme Françoise Gaudet-Smet, ${ }^{22}$ ou bien encore les humbles ménagères et les femmes des grévistes, ce sont également les voix de ces dernières qui confirment l'identité du sujet. Dans une lettre adressée à l'auteure, Gabrielle Roy encourage notamment Simonne Monet-Chartrand à continuer d'écrire pour la radio, car elle juge que celle-ci peut 'réellement faire du bien à beaucoup d'âmes, à beaucoup de pauvres femmes qui se sentent incomprises et qui le sont en fait' $(t$. 3, p. 220). De même, Simonne Monet-Chartrand est invitéeà participer à l'émission 'Fémina', parce qu'il semble indispensable de donner 'aux femmes des notions de psychologie des sexes, de saine alimentation, d'éducation et d'information politique' (t. 3, p. 160). Et Louise 
Simard affirme que sa 'manière d'envisager [son] rôle de maman [...] pourrait servir d'exemple à toutes celles qui sont portées à se décourager en face de leur lourde et ingrate tâche quotidienne' (t. 3, p. 161). La singularité exemplaire du ' je' témoin et les procédés de miroir qu'implique cette position sont également attestés par le 'discours d'escorte'. Dans la Presse, Andrée Lepage souligne 'la lutte que [Simonne Monet-Chartrand] a menée pour avoir le droit de penser par elle-même, à une époque où l'on n'en demandait pas tant aux femmes, et pour poursuivre ses activités sociales et politiques (bénévoles) malgré ses grossesses successives (...) et malgré les réprobations deson entourage' ${ }^{23}$ Andrée Ferreti, qui n'éprouve pas le désir de 'nimber d'une auréole légendaire' Simonne Monet-Chartrand, juge pourtant que son témoignage est exemplaire, puisqu'il révèle un 'sens profond de la justice, [... un] désir immense de paix, [un ...] besoin irrépressible de communication' ${ }^{24}$

Femme d'action, témoin attachant, humain et lucide, Simonne Monet-Chartrand développe dans $M a$ vie comme rivière l' histoire de sa personnalité, c'est-à-dire les figures identificatoires de son passé, l'émergence de son moi social, son combat pour exister en tant que femme, lire, écrire, et étudier tout en étant à l'écoute de 'ce qu['elle] a fait de mieux dans sa Vie' (t. 1, p. 8), ses enfants. L'expression de ce vécu qui prend la forme d'un album ou d'un récit de presse construit avec des documents authentiques traduit le désir de montrer en direct les événements et circonstances qui ont façonné la petite histoire socio-politique de Québec et de constituer par le fait même une mémoire typologique qui permet à chaque membre dese reconnaître: la famille, les nationalistes québécois, les femmes. Traversé par une intention militante, le discours du vécu dans Ma vie comme rivière ne vise pas à endoctriner, ni à susciter l'indignation; bien au contraire, la voix discrète mais omniprésente deSimonne Monet-Chartrand tend à abolir les distances entre le passé et le présent, entre le 'je' raconté et le 'je' racontant, entre le témoin et la collectivité par un témoignage qui rétablit 'par delà cette époque, par delà ses engagements, la permanence des liens avec un passé qui, pour être familial, $n^{\prime}$ en est pas moins le nôtre.' 25 


\section{Notes}

1. Voir à ce sujet les études suivantes:

Terray, L.-M.,'Le discours du vécu,' numéro spécial sur les récits de vie, Revue des sciences humaines,t. 63, no 192, octobre-décembre 1983, p. 7-13. Henry, Chantal, 'Discours d'escorte et promotion publicitaire', numéro spécial sur les récits de vie, Revue des sciences humaines, t. 63, no 192, octobre-décembre 1983, p. 117-124.

2. Monet-Chartrand, Simonne, Ma vie comme rivière, Montréal, Éditions du remue-ménage, 1981, t. 1, 292 p.; 1982, t, 2, 357 p.; 1988, t, 3, 341 p. Désormais les références à cette oeuvre suivront immédiatement les citations.

3. Cléroux, Françoise, 'Le partage des tâches à tous les niveaux: Entrevue avec Simonne Monet-Chartrand,' Féminin-Pluriel, vol. 2, no 3, juin 1982, p. 45.

4. Abastado, Claude, 'Raconte. Raconte!...' Les récits de vies comme objet sémiotique', numéro spécial sur les récits de vie, Revue des sciences humaines, t. 62, no 191, juillet-septembre 1983, p. 19.

5. Cléroux, Françoise, Op. cit., p. 37.

6. Ibid, p. 45.

7. Vercier, Bruno, 'Le mythe du premier souvenir : Pierre Loti, Michel Leiris,' Reoue de l'Histoire littéraire de France, novembre-décembre 1975, p. 1023.

8. Clapier-Valladon, Simone et Poirier, Jean, 'Psychobiographie, Ethnobiographie', numéro spécial sur les récits de vie, Revue des sciences humaines, t. 62, no 191, juillet-septembre 1983, p. 47.

9. Cléroux, Françoise, Op. cit., p. 45.

10. Roy, Monique 'Simonne Monet : la copie non retouchée', le Devoir, 11 décembre 1982, p. 29.

11. Major, Jean-Louis, 'Une fin d'époque. Ma vie comme rivière (tome 2 ) de Simonne Monet-Chartrand', Lettres québécoises, no 29, printemps 1983, p. 55.

12. Ferretti, Andrée, 'Ma vie comme rivière, Un témoignage exemplaire', le Devoir, 20 juin 1981, p. 26.

13. Major, Jean-Louis,Op. cit., p. 57.

14. Garcia, Irma, la Promenade femmilière, Paris, Édition des femmes, 1981, vol. 1, p. 225.

15. Se caractérisant par l'emploi du passé simple de l'imparfait et du conditionnel, le 'monde raconté' est constitué d'écrits tels que la nouvelle et le roman, tandis que le 'monde commenté' marqué essentiellement par le passé composé, le présent et le futur, englobe 'le drame, l'essai biographique, la critique littéraire, le traité philosophique.' Voir l'étude de Harald Weinrich, le Temps, Paris, Seuil, 1973, p. 38. Par ailleurs, dans notre thèse de doctorat 'Le récit autobiographique chez Simonne MonetChartrand, Paule Saint-Onge et Claire Martin,' nous avons étudié la perspective temporelle dans $M a$ vie comme rivière, également à ce sujet notre étude 'Du monde raconté à l'autoportrait: Dans un gant de fer, la Vie défigurée, Ma vie comme rivière', Revue de l'Université de Moncton, vol. 19, nos 2-3, 1986, p. 167-186.

16. Maisani-Léonard Martine, André Gide ou l'ironie de l'écriture, Montréal, Presses de l'Université de Montréal, 1976, p. 20. 
17. Nous avons mis en italiques ces verbes.

18. Lejeune, Philippe, 'Le document vécu,' Je est un autre, Paris, Seuil, 1980, p. 206.

19. Terray, M.-L., 'Le discours du vécu,' Reoue des sciences humaines, t. 63, no 192, octobre-décembre 1983, p. 12.

20. Ducrot, Oswald, Dire et ne pas dire, Paris, Herman, 1980, p. 16.

21. Dans le Scandale du corps parlant (Paris, Seuil, 1980, p. 22), Shoshana Felman résume de façon claire et succinte les différentes catégories de performatifs étudiés par J. L. Austin : les verdictives se rapportent aux 'exercices de jugement (condamner, acquitter, estimer, évaluer, etc.); les excercitives, aux 'assertions d'autorité ou des exercices de pouvoir (commander, donner un ordre, nommer, conseiller, pardonner, etc.)' les commissives, à 'l'engagement vis-à-vis d'une action future (promettre, contracter, épouser, [...] etc.); les behavitives, à une attitude sociale (féliciter, s'excuser, saluer, etc.)'; les expositives, à 'la clarification discursive (affirmer, dénier, interroger, demander, remarquer, etc.)'

22. Dans une lettre adressée à son fiancé (le 30 juillet 1941,t. 2 p. 91-93) et dans les propos qui suivent cette lettre, Simonne Monet-Chartrand traite de la personnalité et des réalisations de Françoise Gaudet-Smet : création de la revue Paysana, organisation d'expositions d'artisanat, de conférences adressées aux femmes, de rencontres prévues avec des groupes de femmes en région rurale.

23. Lepage, Jocelyne, 'Simonne Monet Chartrand à contre-courant,' La Presse, 21 novembre 1982, p. 3, col. 2.

24. Ferreti, Andrée, Op. cit., p. 26.

25. Major, Jean-Louis, Op. cit., p. 57. 